\title{
Transluminal stone removal from right intrahepatic bile duct using novel basket catheter
}

Intrahepatic bile duct (IHBD) stones are usually removed under endoscopic retrograde cholangiopancreatography (ERCP) [1-3]. However, ERCP can be challenging when patients have a complicated surgical anatomy. Various intervention techniques under endoscopic ultrasound (EUS) guidance, such as antegrade stone removal, have been applied [4], but right IHBD stone removal can be difficult owing to device limitations. A novel basket catheter (Reforma; Piolax Medical Devices Inc., Kanagawa, Japan), which can be used for pancreatic stone removal under ERCP guidance [5], has recently become available (\$Fig.1). We describe technical tips for transluminal bile duct stone removal from the right IHBD using this novel catheter ( $\triangleright$ Video 1 ).

A 78-year-old man with obstructive jaundice and right IHBD caused by hepaticojejunostomy stricture was initially treated by EUS-guided hepaticogastrostomy (HGS) using a covered metal stent. A fistula was created and IHBD stone removal was subsequently attempted. Contrast medium was injected after guidewire insertion into the biliary tract through the HGS route. However, cholangiography revealed right IHBD stones and stenosis ( Fig.2a). We therefore dilated the stenosis using a balloon ( $\mathbf{F i g . 2} \mathbf{b}$ ), and then inserted a SPY-DS cholangioscope (Boston Scientific, Tokyo, Japan) into the right IHBD before performing endoscopic hydraulic lithotripsy ( $\triangleright$ Fig.2c). We fragmented and removed the stones using a conventional basket catheter. However, small stones that persisted in the periphery of the right IHBD were removed using the novel basket catheter ( $\mathbf{F i g . 2 d}$ ). A plastic stent was then deployed from the IHBD to the stomach without any adverse events.

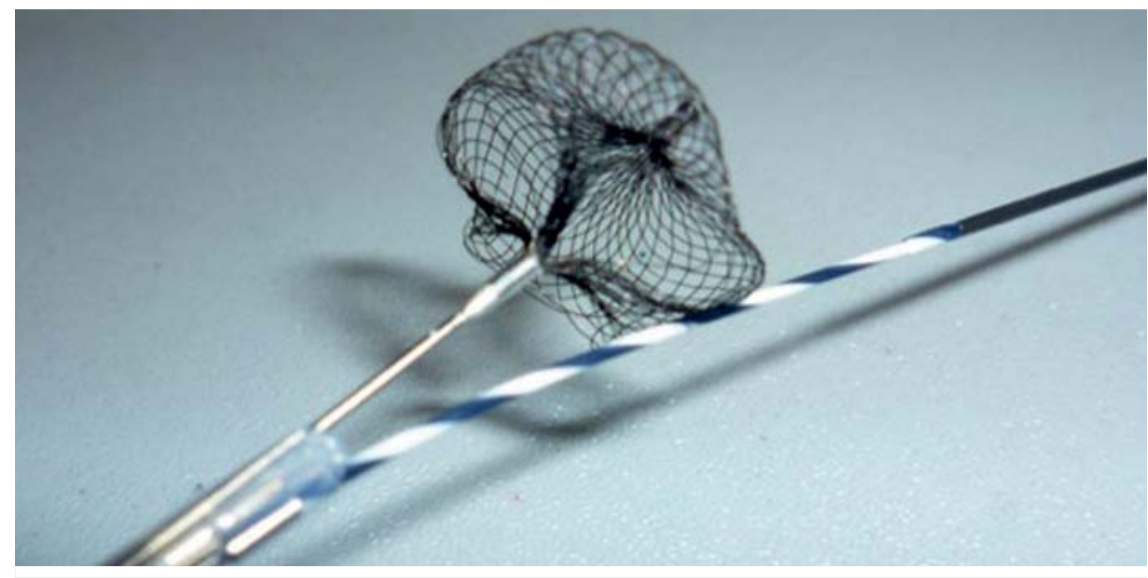

Fig. 1 Novel Reforma basket catheter (Piolax Medical Devices Inc., Kanagawa, Japan).

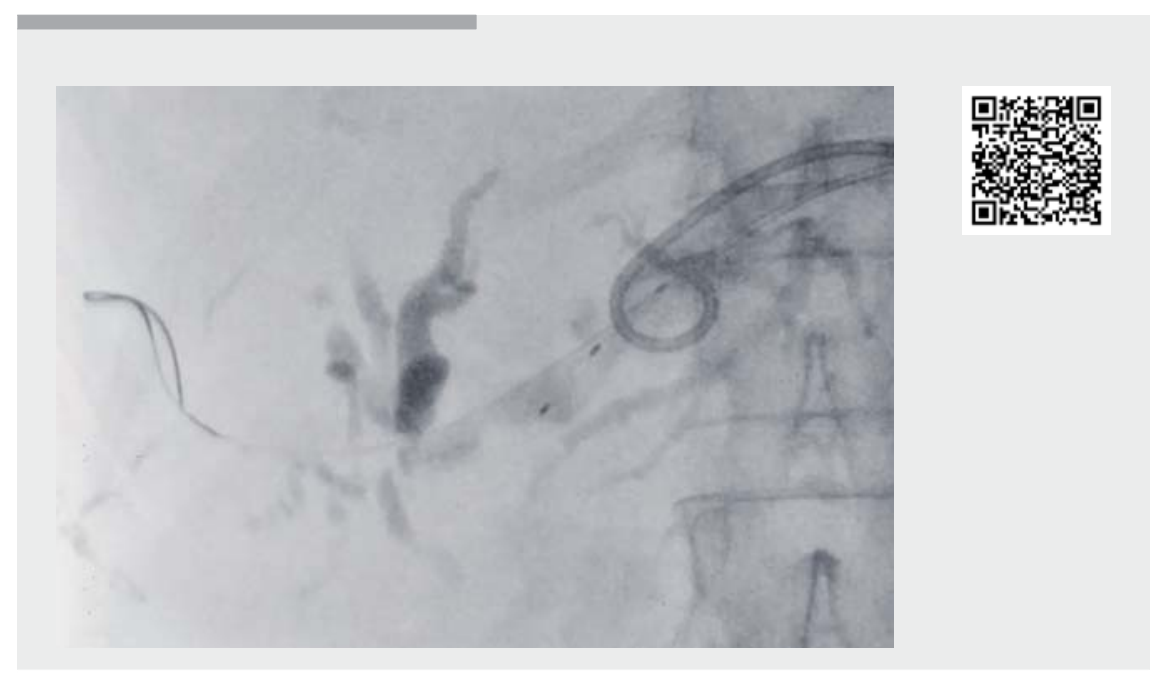

Video 1 Small bile duct stones persisting in the periphery of the intrahepatic bile duct were removed using the novel basket catheter.

The novel basket catheter might be useCompeting interests ful for IHBD stone removal, not only under ERCP guidance but also via the transluminal route.

The authors declare that they have no conflict of interest.

Endoscopy_UCTN_Code_TTT_1AR_2AJ 

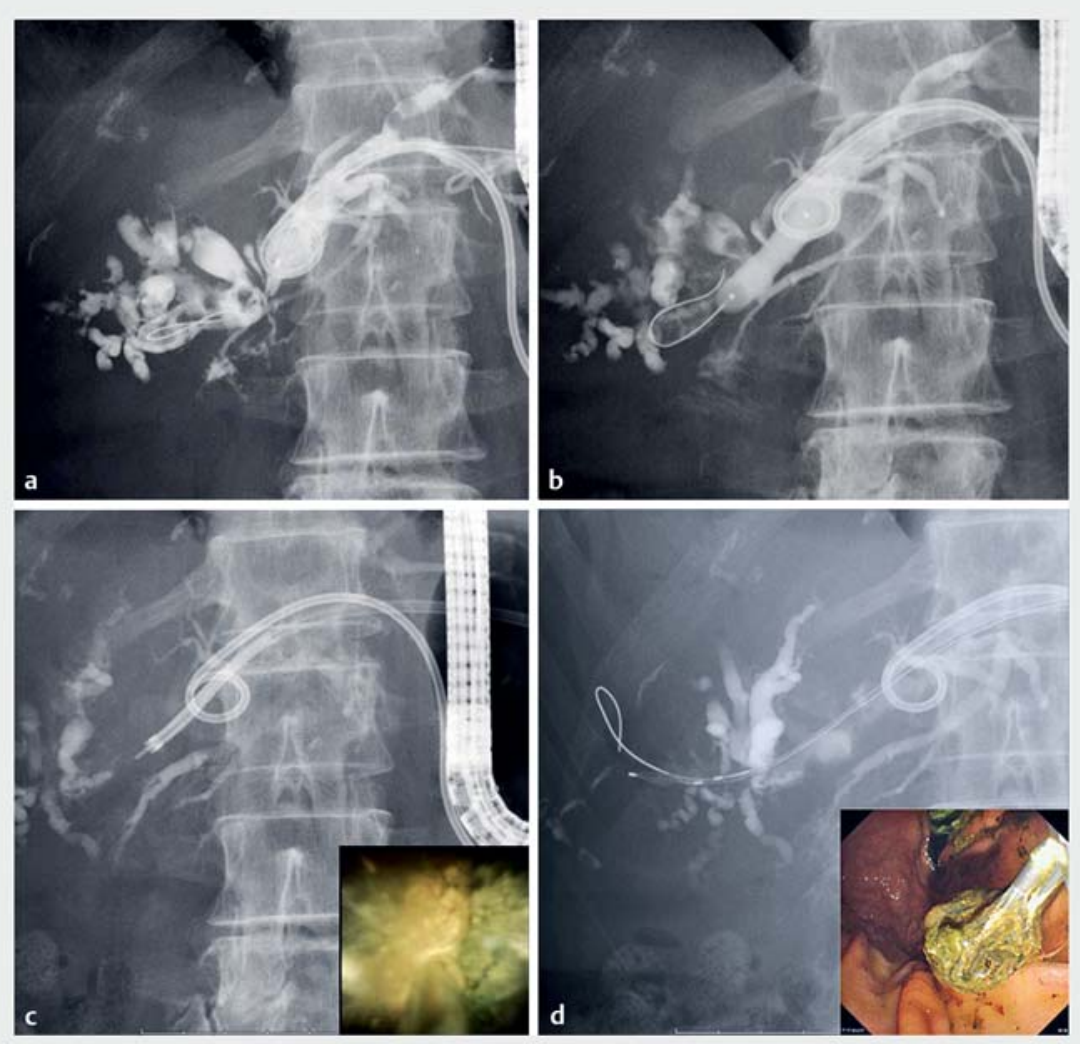

Fig. 2 Cholangiographic images. a Bile duct stones and stenosis were evident in the right intrahepatic bile duct. b The bile duct stenosis was dilated using a balloon catheter. c Endoscopic hydraulic lithotripsy. $\mathbf{d}$ Removal of right intrahepatic bile duct stones using the novel basket catheter.
Bibliography

Dol https://doi.org/10.1055/a-1073-7511

Published online: 20.12.2019

Endoscopy 2020; 52: E223-E224

(C) Georg Thieme Verlag KG

Stuttgart $\cdot$ New York

ISSN 0013-726X

\section{ENDOSCOPY E-VIDEOS \\ https://eref.thieme.de/e-videos}

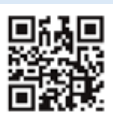

Endoscopy E-Videos is a free access online section, reporting on interesting cases and new techniques in gastroenterological endoscopy. All papers include a high quality video and all contributions are freely accessible online.

This section has its own submission website at https://mc.manuscriptcentral.com/e-videos

\section{The authors}

\section{Takeshi Ogura, Nobu Nishioka, Saori Ueno,} Tadahiro Yamada, Kazuhide Higuchi 2nd Department of Internal Medicine, Osaka Medical College, Osaka, Japan

\section{Corresponding author}

\section{Takeshi Ogura, MD}

2nd Department of Internal Medicine, Osaka Medical College, 2-7 Daigakuchou, Takatsukishi, Osaka 569-8686, Japan Fax: +81-72-6846532

oguratakeshi0411@yahoo.co.jp

\section{References}

[1] Ogura T, Higuchi K. A review of treatment options for bile duct stones. Expert Rev Gastroenterol Hepatol 2016; 10: 1271-1278

[2] Aburajab M, Dua K. Endoscopic management of difficult bile duct stones. Vurr Gastroenterol Rep 2018; 23: 8

[3] Kedia P, Tarnasky PR. Endoscopic management of complex biliary stone disease. Gastrointest Endosc Clin N Am 2019; 29: 257275

[4] Mukai S, Tsuchiya T, Itoi T. Interventional endoscopic ultrasonography for benign biliary disease in patients with surgically altered anatomy. Curr Opin Gastroenterol 2019; 35: 408-415

[5] Sasahira N, Isayama H, Tokyokawa Y et al. A novel basket catheter to facilitate endoscopic removal of pancreatic stones (with video). Gastrointest Endosc 2013; 78: 925929 